\title{
Dietary nitrate supplementation increases acute mountain sickness severity and sense of effort during hypoxic exercise
}

Article

Accepted Version

Rossetti, G. M. K. ORCID: https://orcid.org/0000-0002-96106066, Macdonald, J. H., Wylie, L. J., Little, S. J., Newton, V., Wood, B., Hawkins, K. A., Beddoe, R., Davies, H. E. and Oliver, S. J. (2017) Dietary nitrate supplementation increases acute mountain sickness severity and sense of effort during hypoxic exercise. Journal of applied physiology., 123 (4). pp. 983-992. ISSN 1522-1601 doi:

https://doi.org/10.1152/japplphysiol.00293.2017 Available at https://centaur.reading.ac.uk/97686/

It is advisable to refer to the publisher's version if you intend to cite from the work. See Guidance on citing.

To link to this article DOI: http://dx.doi.org/10.1152/japplphysiol.00293.2017

Publisher: American Physiological Society

All outputs in CentAUR are protected by Intellectual Property Rights law, including copyright law. Copyright and IPR is retained by the creators or other copyright holders. Terms and conditions for use of this material are defined in the End User Agreement. 


\section{www.reading.ac.uk/centaur}

\section{CentAUR}

Central Archive at the University of Reading

Reading's research outputs online 
1 Dietary nitrate supplementation increases acute mountain sickness severity and sense of effort during

2 hypoxic exercise

3

4

5 Gabriella M.K. Rossetti ${ }^{1}$, Jamie H. Macdonald ${ }^{1}$, Lee J. Wylie ${ }^{2}$, Samuel J. Little ${ }^{1}$, Victoria Newton ${ }^{1}$, Benjamin

6 Wood $^{1}$, Kieran A. Hawkins ${ }^{1}$, Rhys Beddoe ${ }^{1}$, Hannah E. Davies ${ }^{1}$, and Samuel J. Oliver ${ }^{1}$.

7

8

$9 \quad{ }^{1}$ Extremes Research Group, College of Health and Behavioural Sciences, Bangor University, Bangor, Gwynedd,

10 United Kingdom;

$11{ }^{2}$ Sport and Health Sciences, College of Life and Environmental Sciences, University of Exeter, St. Luke's

12 Campus, Exeter, United Kingdom

13

14

15 Running head: Nitrate supplementation increases AMS severity

16

17

18 Address for correspondence:

19 Samuel J. Oliver PhD

20 College of Health and Behavioural Sciences,

21 Bangor University,

22 Bangor,

23 LL57 2PZ,

24 UK.

25 Email: s.j.oliver@bangor.ac.uk

26 Telephone: +44 1248383965 
Dietary nitrate supplementation enhances sea level performance and may ameliorate hypoxemia at high altitude. However, nitrate may exacerbate acute mountain sickness (AMS), specifically headache. This study investigated the effect of nitrate supplementation on AMS symptoms and exercise responses with 6h hypoxia. Twenty recreationally-active men (mean(SD): age 22(4) years, $\dot{\mathrm{V}} \mathrm{O}_{2 \max } 51(6) \mathrm{mL} \cdot \mathrm{min}^{-1} \cdot \mathrm{kg}^{-1}$ ) completed this randomized double-blinded placebo-controlled crossover study. Twelve participants were classified as AMS- based on Environmental Symptom Questionnaire (AMS-C) score $<0.7$ in both trials, and five participants were classified as $\mathrm{AMS}+$ based on AMS-C score $\geq 0.7$ on placebo. Five days nitrate supplementation ( $70 \mathrm{~mL}$ beetroot juice containing $\sim 6.4 \mathrm{mmol}$ nitrate daily) increased plasma NO metabolites by $182 \mu \mathrm{M}$ compared to placebo but did not reduce AMS or improve exercise performance. After 4 h hypoxia $\left(\mathrm{F}_{\mathrm{I}} \mathrm{O}_{2}=0.124\right)$ nitrate increased AMS-C and headache severity (visual analogue scale (VAS); whole sample $\Delta 10[1,20] \mathrm{mm} ; p=0.03$ ) compared to placebo. In addition, after $5 \mathrm{~h}$ hypoxia, nitrate increased sense of effort during submaximal exercise $(\Delta 7[-1,14] ; p=0.07)$. In AMS- nitrate did not alter headache or sense of effort. In contrast, in AMS+ nitrate increased headache severity $(\Delta 26[-3,56] \mathrm{mm} ; p=0.07)$, sense of effort $(\Delta 14[1,28] ; p=0.04)$, oxygen consumption, ventilation, and mean arterial pressure during submaximal exercise. On the next day, in a separate acute hypoxic exercise test $\left(\mathrm{F}_{\mathrm{I}} \mathrm{O}_{2}=0.141\right)$, nitrate did not improve time to exhaustion at $80 \%$ hypoxic $\dot{\mathrm{V}}_{2 \max }$. In conclusion, dietary nitrate increases AMS and sense of effort during exercise, particularly in those who experienced AMS. Dietary nitrate is therefore not recommended as an AMS prophylactic or ergogenic aid non-acclimatized individuals at altitude.

KEY WORDS: Altitude sickness, beetroot, headache, nitric oxide, rating of perceived exertion

\section{NEW AND NOTEWORTHY}

This is the first study to identify that the popular dietary nitrate supplement (beetroot) does not reduce acute mountain sickness (AMS) or improve exercise performance during $6 \mathrm{~h}$ hypoxia. The consumption of nitrate in those susceptible to AMS exacerbates AMS symptoms (headache) and sense of effort, and raises oxygen cost, ventilation, and blood pressure during walking exercise in 6 h hypoxia. These data question the suitability of nitrate supplementation during altitude travel in non-acclimatized people. 


\section{INTRODUCTION}

Many people engage in altitude travel for work and leisure, with 300 million overnight stays in alpine regions each year (5) and hundreds of thousands of individuals traveling to other high altitude regions such as the Himalayas and Kilimanjaro (17). Approximately half of those that visit high altitude suffer from illnesses such as acute mountain sickness (AMS) (6). AMS can lead to high altitude cerebral edema (HACE) which if left untreated can be fatal (19). High altitude exposure also reduces exercise capacity (16). Finding interventions to counteract hypoxemia, the root-cause of these negative effects, is therefore important.

Nitric oxide (NO) is a potent signaling molecule that modulates human physiological function via its role in the regulation of blood flow, muscle contractility and mitochondrial respiration (44). NO can be produced via the oxygen $\left(\mathrm{O}_{2}\right)$-dependent oxidation of L-arginine with the help of NO synthase (NOS) enzymes (31). However, it is now clear that NO can also be generated by the serial reduction of nitrate to nitrite and then NO (12). An increase in NO bioavailability has been observed after the consumption of dietary inorganic nitrate (49) and has been associated at sea level with a reduction in resting blood pressure (43), increased skeletal muscle blood flow (14), and enhanced exercise performance (21). Importantly, the reduction of nitrite to NO is increased in environments of low $\mathrm{O}_{2}$ tension, and therefore dietary nitrate consumption may be a particularly effective method to increase NO bioavailability at altitude. During acute exposure to hypoxia $(\leq 2 \mathrm{~h})$, nitrate supplementation has been shown to improve peripheral and muscle oxygenation during rest and exercise (33), and improve muscle energetics (46), lower the $\mathrm{O}_{2}$ cost of submaximal exercise (24), and enhance exercise performance in some (e.g. $(33,42))$ but not all (e.g. $(2,10))$ studies. NO may also improve diffusion capacity within the lung, and enhance $\mathrm{O}_{2}$ delivery (15), potentially ameliorating the root cause of AMS (hypoxemia). Because of these proposed beneficial physiological and exercise responses, some have recommended dietary nitrate for altitude travel (4, 37), with a recent study concluding its use is safe and feasible at altitude (20).

However, nitrate supplementation may be harmful during hypoxia. Dietary nitrate may increase AMS by elevating headache. Not only the cardinal symptom of AMS, it has been suggested that high-altitude headache contributes to the accompanying AMS symptoms of gastrointestinal symptoms, fatigue, dizziness, lightheadedness, and poor sleep (27). A growing body of data supports the proposed pathophysiology of high-altitude headache as a result of arterial dilation causing elevated cerebral blood volume and intracranial pressure. This leads to increased arterial and intracranial pressure transmission, trigeminovascular sensitization, and pain sensation (27). Given that 
84 NO is implicated in hypoxia-induced vasodilation (35), and directly stimulates the trigeminovascular system (3),

85 supplementing with nitrate may exacerbate high-altitude headache pathophysiology. However, to date no laboratory studies have been completed to investigate the effects of dietary nitrate on AMS. Resolving the efficacy and safety of this dietary intervention, particularly in reference to the cerebral component of AMS, is of clinical and timely importance. In addition, the effect of dietary nitrate consumption on exercise responses during longerduration hypoxic exposures $(>2 \mathrm{~h})$ is unknown. Since the physiological stimulus of exercise may increase AMS severity in hypoxia $(11,38)$, the effects of nitrate on exercise responses, whether positive or negative, could potentially alter nitrate's effect on AMS symptoms. Given that individuals travelling to high altitude will engage in exercise, it is important to investigate the effects of nitrate under conditions of exercise in hypoxia. In addition, AMS is a condition that is greatly affected by individual susceptibility (48). It is possible that nitrate may exacerbate AMS pathophysiology and symptoms in individuals who experience AMS, but have beneficial physiological and exercise performance effects in individuals who do not suffer AMS.

The primary aim of the present double-blind placebo-controlled crossover study was to determine the effect of five days of dietary nitrate supplementation on high-altitude headache and AMS symptom severity with a $6 \mathrm{~h}$ exposure to hypoxia. We hypothesized five days of dietary nitrate supplementation would decrease high-altitude headache and AMS symptom severity with a 6 h exposure to hypoxia. Secondary aims of the present study were to explore: the effect of five days dietary nitrate supplementation on submaximal exercise responses during $6 \mathrm{~h}$ hypoxia; and the interaction of AMS (AMS present or absent) and nitrate supplementation on high-altitude headache severity and submaximal exercise responses during $6 \mathrm{~h}$ hypoxia. In addition, due to equivocal findings in the literature (e.g. $(10,33)$ ), we also determined the effect of six days dietary nitrate supplementation on exercise performance in acute hypoxia. 
Twenty recreationally-active men were recruited into the study (mean (SD); age, 22 (4) years; height, 180 (10) cm; body mass, $79(11) \mathrm{kg}$; $\left.\dot{\mathrm{VO}}_{2 \max }, 51(6) \mathrm{ml} \cdot \mathrm{kg}^{-1} \cdot \mathrm{min}^{-1}\right)$. Eleven participants $(45 \%)$ had previously travelled to high altitude ( $\geq 2500 \mathrm{~m}$ ), and of these 11 participants, 2 (18\%) reported previous AMS, and none had a history of HAPE or HACE. Participants had not travelled to altitude $(\geq 1500 \mathrm{~m})$ in the preceding six months, and had no medical contraindications to maximal exercise testing. All participants provided written informed consent. Ethical approval was granted by the Ethics Committee of the School of Sport, Health, and Exercise Sciences at Bangor University, and the study was registered on www.clinicaltrials.gov (trial ID: NCT03101904).

Study design

The study followed a double-blinded placebo-controlled crossover design. Normoxic $\left(\mathrm{F}_{\mathrm{l}} \mathrm{O}_{2}=0.209\right.$; sea level $)$ and hypoxic $\left(\mathrm{F}_{\mathrm{I}} \mathrm{O}_{2}=0.141\right.$; equivalent $\left.3225 \mathrm{~m}\right)$ maximal exercise tests were conducted at baseline. Participants then completed two six-day supplementation periods, separated by a minimum ten-day washout. On day five of each supplementation period, a $6 \mathrm{~h}$ hypoxic exposure $\left(\mathrm{F}_{\mathrm{I}} \mathrm{O}_{2}=0.124\right.$; equivalent $\left.4219 \mathrm{~m}\right)$ was conducted to assess AMS and responses to submaximal exercise. On day six, a time to exhaustion test was conducted at $80 \% \dot{\mathrm{VO}}_{2 \max }$ reserve in acute hypoxia $\left(\mathrm{F}_{\mathrm{I}} \mathrm{O}_{2}=0.141\right.$; equivalent $\left.3225 \mathrm{~m}\right)$ to assess exercise performance. An overview of the protocol is depicted in Figure 1.

\section{Supplementation}

Participants were randomly assigned to receive six days of inorganic nitrate or placebo supplementation, separated by a minimum ten-day washout. Supplementation consisted of a daily nitrate-rich concentrated beetroot juice shot (nitrate; $70 \mathrm{~mL} /$ day containing $\sim 6.4 \mathrm{mmol} \mathrm{NO}_{3}^{-}$; Beet It Sport, James White drinks Ltd, Ipswich, UK) or a nitratedepleted concentrated beetroot juice shot (placebo; $70 \mathrm{~mL} /$ day containing $\sim 0.003 \mathrm{mmol} \mathrm{NO}_{3}{ }^{-}$; James White drinks Ltd, Ipswich, UK) that was identical in appearance, taste, and texture. Placebo shots were created by passing the $\mathrm{NO}_{3}{ }^{-}$rich concentrated beetroot juice through a Purolite $\mathrm{A} 520 \mathrm{E} \mathrm{NO}_{3}{ }^{-}$selective ion exchange resin before pasteurization (26). Supplements were ingested $2.5 \mathrm{~h}$ before any experimental tests, and at the same time each day throughout both supplementation periods. Participants were phoned or texted to remind them to ingest the supplement and were asked to confirm ingestion by phone or return text. 
To isolate nutritional effects of the intervention, participants avoided high $\mathrm{NO}_{3}{ }^{-}$food and drink throughout the study, and diet and physical activity was matched before each trial, confirmed by food and exercise diaries. Participants also abstained from using antibacterial mouthwashes as this has previously shown to lessen the reduction of $\mathrm{NO}_{3}{ }^{-}$to $\mathrm{NO}_{2}^{-}$by commensal bacteria within the oral cavity (18).

Before (day 0) and on day five (in hypoxia) and day six (pre-hypoxia) of each supplementation phase, venous blood samples were collected into lithium heparin-coated $6 \mathrm{~mL}$ vacutainers (BD Vacutainer tubes; Becton, Dickinson and Company: New Jersey), immediately centrifuged at $4000 \mathrm{rpm}$ and $4^{\circ} \mathrm{C}$ for 7 min before the plasma was extracted and stored at $-80^{\circ} \mathrm{C}$ for later analysis of plasma $\mathrm{NO}$ metabolites (nitrate + nitrite $[\mathrm{NOx}]$ ). To confirm participants were blind to the supplementation they were receiving and that the placebo was successful, a manipulation check was conducted after each supplementation was completed, by asking participants to guess what intervention they had received (possible responses were "beetroot”, "placebo", or "don’t know”).

Procedures

Maximal aerobic capacity $\left(\dot{\mathrm{VO}}_{2 \max }\right)$

All exercise tests consisted of loaded walking whilst carrying a $15 \mathrm{~kg}$ rucksack on a motorized treadmill (H-PCosmos, Sports \& Medical GmbH; Nussdorf-Traunstein: Germany). At baseline, participants completed an incremental exercise test to exhaustion with simultaneous online pulmonary gas analysis (Cortex Metalyzer, Biophysik GmbH; Leipzig: Germany). The incremental exercise test protocol commenced at $5 \mathrm{~km} / \mathrm{h}$ and $1 \%$ gradient with a ramped increase in gradient to $25 \%$ over 18 min. If $25 \%$ was reached, treadmill speed was increased by $0.66 \mathrm{~km} / \mathrm{h} / \mathrm{min}$. Sense of effort during exercise (Rating of Perceived Exertion; RPE) was recorded each minute of the test using the Borg CR100 (9). $\dot{\mathrm{VO}}_{2 \max }$ was identified if all of the following criteria were met: volitional fatigue; a plateau in oxygen consumption $\left(\mathrm{V}_{2}\right)$ despite an increase in workload; respiratory exchange ratio $(\mathrm{RER}) \geq 1.15$. Participants also completed the same maximal exercise protocol in hypoxia $\left(\mathrm{F}_{\mathrm{I}} \mathrm{O}_{2}=0.141\right.$, equivalent $3225 \mathrm{~m}$ ), with the two tests separated by a minimum of $48 \mathrm{~h}$.

$\underline{\text { Six-hour hypoxic exposure }}$

163 On day five of each supplementation period, participants completed a six-hour poikilocapnic hypoxic exposure in a normobaric hypoxic chamber $\left(\mathrm{F}_{\mathrm{I}} \mathrm{O}_{2}=0.124\right.$; equivalent 4219 m: Hypoxico, Inc. New York, NY). After fifteen min seated rest, participants completed the first of three 20 -min bouts of submaximal exercise at $40 \%$ hypoxic 
$166 \dot{\mathrm{V}}_{2 \max }$ reserve. The submaximal exercise protocol during the $6 \mathrm{~h}$ exposure consisted of exercise bout one, 167 completed between 15-35 min; exercise bout two, 140-160 min; and exercise bout three, 300-320 min. Blood 168 pressure and heart rate measured by a stress BP system (Tango+; SunTech Medical, Inc., Morrisville, NC; USA), and oxygen saturation $\left(\mathrm{SpO}_{2}\right)$ measured by pulse oximetry (9550 OnyxII; Nonin Medical Inc, Minnesota), were assessed every hour and pre- and post-each exercise bout throughout the $6 \mathrm{~h}$ exposure. Mean arterial blood pressure (MAP) was calculated to account for heart rate using the equation provided by Moran and colleagues (36):

$$
\mathrm{MAP}=\mathrm{DBP}+((0.01 \times \exp (4.14-40.74 \div \mathrm{HR})) \times(\mathrm{SBP}-\mathrm{DBP}))
$$

Where MAP, Mean arterial blood pressure; DBP, Diastolic blood pressure; HR, Heart rate; SBP, Systolic blood pressure.

\section{High-altitude headache and AMS}

High-altitude headache was assessed every hour and pre- and post-each exercise bout throughout the six-hour exposure on day five of supplementation, using a 0-100 mm visual analogue scale (VAS) (23). Participants were asked to mark on the line the intensity/feeling that corresponded to their headache, where the beginning of the line indicated no perception/feeling at all and the end of the line indicated maximum perception/feeling. AMS was assessed by the Acute Cerebral Mountain Sickness (AMS-C) score calculated from the 11-item Environmental Symptoms Questionnaire (ESQ) (41). Participants rated the severity of each item from one to five, and the ratings were multiplied by their factorial loadings and summed. In order to investigate whether any effects of nitrate supplementation were affected by the presence or absence of AMS, participants were split into two groups depending on whether they had AMS on placebo. Participants with AMS-C $<0.7$ in both trials were classified as AMS-, while participants with AMS-C $\geq 0.7$ on placebo were classified as AMS+ (40). Participants with AMS$\mathrm{C}<0.7$ on placebo, but AMS-C $\geq 0.7$ on nitrate were included in the whole sample analyses, but were not included in AMS+/- sub-analyses.

\section{Submaximal exercise responses}

Sense of effort during the three submaximal exercise bouts was assessed by RPE. RPE was recorded using the Borg CR100 (9) which asks participants to rate the intensity of the exercise sensation using numbers from 0-100+ and verbal descriptors (e.g. "moderate", equivalent to 25). Extensive evidence supports the use of RPE as a valid and appropriate method to record sense of effort and perceptual responses to exercise (13). RPE, heart rate and 
$\mathrm{SpO}_{2}$ were recorded at two-min intervals during the three exercise bouts. In addition to these variables, the study

197

198

199

200

201

202

203

204

205

206

207

208

209

210

211

212

213

214

215

216

217

218

219

220

221

222

223

224

225

design targeted exercise bout two to examine extra physiological responses, including ventilatory parameters and exercising blood pressure, in an attempt to identify possible mechanisms of nitrate's effect on exercise and AMS before AMS was present. We did not collect this data during exercise bout one because this bout occurred too early, when variable responses such as the hypoxic ventilatory response (47) would likely mask any mechanistic physiological changes. We did not collect this data during exercise bout three because this bout occurred too late, when AMS was already present. To be consistent with a cause and effect relationship, any physiological changes must be observed before the onset of AMS; at exercise bout three it would not be possible to differentiate between cause and consequence of AMS. Thus, during exercise bout two exercising blood pressure was recorded every two min and ventilation $\left(\mathrm{V}_{\mathrm{E}}\right), \dot{\mathrm{V}} \mathrm{O}_{2}, \mathrm{RER}$, and end-tidal carbon dioxide $\left(\mathrm{P}_{\mathrm{ET}} \mathrm{CO}_{2}\right)$ were measured using online gas analysis. To account for the hypoxic conditions, the gas analyzer was calibrated using ambient air $\left(12.4 \% \mathrm{O}_{2}\right.$; $\left.0.03 \% \mathrm{CO}_{2}\right)$ and a $\mathrm{FiO}_{2}$-specific calibration gas $\left(8 \% \mathrm{O}_{2} ; 5 \% \mathrm{CO}_{2}\right.$; Industrial Gases, BOC ltd, Surrey, UK).

\section{Exercise performance (time to exhaustion; TTE)}

On day six of each supplementation period, participants completed a time to exhaustion (TTE) test at $5 \mathrm{~km} / \mathrm{h}$ and a gradient corresponding to $80 \%$ of their hypoxic $\dot{\mathrm{V}} \mathrm{O}_{2 \max }$ reserve in acute hypoxia $\left(\mathrm{F}_{1} \mathrm{O}_{2}=0.141\right.$, equivalent 3225 m). Exercise performance was defined as TTE determined by the time from onset of test to task failure (volitional exhaustion or inability to maintain treadmill speed). During the exercise test $\mathrm{SpO}_{2}$, heart rate, and RPE were recorded each minute. Participants were blind to time elapsed and provided no verbal encouragement.

\section{Plasma nitric oxide metabolites $\left(N O_{x}\right)$}

All glassware, utensils, and surfaces were rinsed with deionised water to remove residue $\mathrm{NO}_{3}{ }^{-}$and $\mathrm{NO}_{2}{ }^{-}$before blood analysis. After thawing at room temperature, plasma samples were initially deproteinized using cold ethanol precipitation. Initially $0.5 \mathrm{~mL}$ of sample was placed in a chilled microcentrifuge tube, along with $1 \mathrm{~mL}$ of cold $\left(0^{\circ} \mathrm{C}\right)$ ethanol; then the samples were vortexed and left to stand at $0^{\circ} \mathrm{C}$ for $30 \mathrm{~min}$. Thereafter, samples were centrifuged at 14,000 rpm for $5 \mathrm{~min}$, and the supernatant removed for subsequent analysis. NOx (nitrate + nitrite) in the deproteinized plasma samples was reduced to NO in the presence of $0.8 \%(\mathrm{w} / \mathrm{v})$ vanadium trichloride in 1 M hydrochloric acid. The production of NO was detected by a Sievers gas-phase chemiluminescence NO analyser (Sievers NOA 280i; Analytix, Duham, UK) and the NOx concentration was derived by plotting signal (mV) area against a calibration plot of $1-750 \mu \mathrm{M}$ sodium $\mathrm{NO}_{3}{ }^{-}$. 
Statistical analyses

228 Differences between nitrate and placebo were determined by confidence intervals relating to a priori meaningful differences (22), supported by statistical differences testing by repeated measures analysis of variance (RM ANOVA) or t-tests as appropriate $(\mathrm{p}<0.05)$. For the primary analysis, (to determine the effect of dietary nitrate supplementation on high-altitude headache) a $2 \times 4$ (Trial $\times$ Time) RM ANOVA was used to compare highaltitude headache severity by VAS from 4 to $6 \mathrm{~h}$ between nitrate and placebo trials. The time course of 4 to $6 \mathrm{~h}$ was chosen for the primary analysis based on the expected time that AMS would develop from previous data from our laboratory at a similar $\mathrm{FiO}_{2}(28-30)$ that showed no incidence of AMS before $4 \mathrm{~h}$, but an AMS incidence of $50 \%$ after $6 \mathrm{~h}$. A sample size estimation for the primary analysis indicated that 16 participants were needed to produce an $80 \%$ chance of obtaining statistical significance at the 0.05 level for a two-tailed design (45), based on a minimum important difference of $10 \mathrm{~mm}(23)$, a standard deviation of the difference of $18 \mathrm{~mm}$, and an estimated average correlation of 0.4 (data from (29)). To determine the influence of AMS, all analyses were repeated including a factor for AMS presence or absence (AMS+ or AMS-), and interpreted on the basis of significant interactions.

The effect of nitrate supplementation on exercise performance (TTE) was determined by paired samples t-test. A sample size estimation for this analysis indicated that 12 participants were needed to produce an $80 \%$ chance of obtaining statistical significance at the 0.05 level for a two-tailed design (8), based on a minimum important difference of $30 \mathrm{~s}$, and a standard deviation of the difference of $33 \mathrm{~s}$ (data from (33)). To determine the effect of nitrate supplementation on i) $\mathrm{RPE}$, ii) $\mathrm{SpO}_{2}$, and ii) heart rate, a $2 \times 5$ (Trial $\times$ Isotime) $\mathrm{RM}$ ANOVA was used to compare nitrate and placebo trials at $0 \%, 25 \%, 50 \%, 75 \%$, and $100 \%$ of isotime during the TTE test. Resting values taken immediately before commencing the TTE were recorded as $0 \%$ isotime. $100 \%$ isotime was defined as the last complete minute of the shortest TTE, and the corresponding minute in the longest TTE for each participant. The minute identified as $100 \%$ isotime was multiplied by $0.25,0.5$, and 0.75 and rounded to the nearest complete minute to give $25 \%$ isotime, $50 \%$ isotime, and $75 \%$ isotime, respectively.

Five participants did not complete the TTE on day six due to injury or technical reasons, e.g. cramp or tripping whilst on the treadmill, and one of these was also removed from analyses relating to RPE on day five due to failure to use the RPE scale consistently. All analyses were completed using SPSS version 23 (IBM Corp, Armonk; NY). 
Participants were sufficiently blinded to the intervention since the manipulation check indicated participants guessed correctly in only $18 \%$ of trials, guessed incorrectly in $34 \%$ of trials, and were unable to distinguish between interventions in $48 \%$ of trials.

Plasma nitric oxide metabolites $\left(N O_{x}\right)$

Subject compliance with the supplementation protocol was $100 \%$. This self-reported compliance measure was confirmed by plasma $\mathrm{NO}_{\mathrm{x}}$ data. Plasma $\mathrm{NO}_{\mathrm{x}}$ was similar between trials at baseline (nitrate $=27$ (9); placebo $=28$ (9); $[-5,3] \mu \mathrm{M} ; p=0.7)$. The nitrate supplementation effectively altered plasma $\mathrm{NO}_{\mathrm{x}}$ concentrations. Compared to placebo, nitrate supplementation increased plasma $\mathrm{NO}_{\mathrm{x}}$ on day five (mean diff $[95 \% \mathrm{CI}]: \Delta 182[155,208] \mu \mathrm{M}$; $p<0.001)$. Compared to placebo, nitrate supplementation also increased plasma $\mathrm{NO}_{\mathrm{x}}$ on day six in the 15 participants that completed the time to exhaustion tests $(\Delta 244[201,286] \mu \mathrm{M} ; p<0.001)$. By design, plasma $\mathrm{NO}_{\mathrm{x}}$ was not altered compared to baseline, with five $(\Delta-3[-8,2] \mu \mathrm{M} ; \mathrm{p}=0.3)$ or six $(\Delta-3[-10,1] \mu \mathrm{M} ; p=0.2)$ days of placebo supplementation.

High-altitude headache and AMS

272 There was no headache at $0 \mathrm{~h}$ (pre-hypoxia) in either trial (nitrate $=1(4)$; placebo $=4(9) ;[-7,2] \mathrm{mm} ; p=0.2$ ). Although headache tended to increase after exercise bout one $(\Delta 4[-1,9] \mathrm{mm} ; p=0.08)$ and bout two $(\Delta 8[0,15]$ $\mathrm{mm} ; p=0.05$ ), there was no effect of nitrate (both $p \geq 0.4$; Figure 2A). As expected, AMS and headache increased in the latter part of the trial $(\mathrm{p}<0.05)$. Unexpectedly this effect was exacerbated by nitrate. From 4 to $6 \mathrm{~h}$, nitrate increased headache severity compared to placebo $(\Delta 10[1,20] \mathrm{mm} ; p=0.03)$ and tended to increase AMS-C compared to placebo $(\Delta 0.15[-0.01,0.31] ; p=0.07$; Figure 2B $)$.

Physiological responses to 6 h hypoxia

280 Resting physiological responses (heart rate, $\mathrm{SpO}_{2}$, and blood pressure) changed throughout the $6 \mathrm{~h}$ exposure, but were not affected by nitrate. Heart rate increased over the hypoxic exposure $(\Delta 16[11,21] \mathrm{bpm} ; p<0.001)$, and after each exercise bout, but was not affected by nitrate $(\Delta-2[-5,2]$ bpm; $p=0.4)$. $\mathrm{SpO}_{2}$ decreased over the hypoxic exposure $(\Delta-14[-17,-11] \% ; p<0.001)$, and after each exercise bout, but was not affected by nitrate $(\Delta 0$ $[-1,1] \% ; p=0.8)$. Nitrate supplementation had no effect on any measure of resting blood pressure, with no 
difference in systolic blood pressure (SBP; $\Delta 1[-4,5] \mathrm{mmHg} ; p=0.7)$, diastolic blood pressure (DBP; $\Delta-1[-4,2]$ $\mathrm{mmHg} ; p=0.4$ ), or mean arterial blood pressure (MAP; $\Delta 1[-3,4] \mathrm{mmHg} ; p=0.8$ ).

\section{Submaximal exercise responses}

Dietary nitrate increased sense of effort compared to placebo during submaximal exercise in 6 h hypoxia (Figure 3A). Nitrate elicited a small but significant increase in RPE for exercise bout one $(\Delta 4[0,8] ; p=0.03)$, and a larger increase for exercise bout three $(\Delta 7[-1,14] ; p=0.07)$, although had no effect on RPE for exercise bout two $(\Delta 2$ $[-5,9] ; p=0.5)$. In the nitrate trial, headache at $5 \mathrm{~h}$ (pre-exercise bout three) was positively correlated with change in RPE from exercise bout one to exercise bout three $(r=0.67 ; p<0.01)$. When analyzed across the whole sample, nitrate had no effect on any physiological response to the three submaximal exercise bouts (Table 1).

\section{Influence of AMS presence or absence (AMS+/-)}

Twelve participants were classified as AMS- and five were AMS+ (see Methods: High-altitude headache and AMS). There were no differences in baseline characteristics or $\mathrm{NO}_{\mathrm{x}}$ concentrations at any point between AMS+ and AMS- (all $p>0.5$; data not shown).

In AMS-, nitrate had no effect on high-altitude headache $(\Delta 2[-5,9] \mathrm{mm} ; p=0.6$; Figure $2 \mathrm{C})$ or AMS-C $(\Delta-0.04$ $[-0.13,0.06] ; p=0.4$; Figure 2D). In AMS+, nitrate increased altitude illness severity from 4 h: in AMS+, highaltitude headache severity was more than doubled with nitrate compared to placebo $(\Delta 26[-3,56] \mathrm{mm} ; p=0.07$; Figure 2E) and AMS-C was similarly increased $(\Delta 0.46[-0.10,1.02] ; p=0.09$; Figure $2 \mathrm{~F})$. In AMS-, there was no difference in RPE between nitrate and placebo for any of the exercise bouts (Figure 3C). In AMS+, by the end of the exposure, dietary nitrate had increased sense of effort during submaximal exercise compared to placebo (Figure $3 \mathrm{E} ; \Delta 14[1,28] ; p=0.04)$.

Depending on whether participants were AMS- or AMS+, nitrate had opposite effects on physiological responses to submaximal exercise during the hypoxic exposure (determined by significant AMS-trial interactions). During exercise bout two, in AMS-, nitrate decreased ventilation in comparison to placebo $(-3.0[-5.5,-0.4] \mathrm{L} / \mathrm{min} ; p=$ 0.03; Figure 4A), and had no effect on $\dot{\mathrm{VO}}_{2}(\Delta-0.02[-0.13,0.09] \mathrm{L} / \mathrm{min} ; p=0.6$; Figure 5A) or MAP $(\Delta-1[-9,6]$ $\mathrm{mmHg} ; p=0.7$; Figure 6A). In contrast, in AMS+, nitrate increased ventilation $(\Delta 3.1[-0.7,7.0] \mathrm{L} / \mathrm{min} ; p=0.1$; Figure 4B), $\dot{\mathrm{VO}}_{2}(\Delta 0.10[0.03,0.17] \mathrm{L} / \mathrm{min} ; p=0.02$; Figure $5 \mathrm{~B})$, and $\mathrm{MAP}(\Delta 7[-3,17] \mathrm{mmHg} ; p=0.1$; Figure 
315 6B). During exercise bout three, in AMS- nitrate increased $\mathrm{SpO}_{2}$ compared to placebo from 12 min onwards ( $\Delta 3$ $316[0,4] \% ; p=0.03$; Figure 3D). However, in $\mathrm{AMS}+$, nitrate had no effect on $\mathrm{SpO}_{2}$ for any exercise bout (Figure

$3173 \mathrm{~F}$ ). The effect of nitrate on $\mathrm{P}_{\mathrm{ET}} \mathrm{CO}_{2}$ was not altered depending on the presence or absence of AMS (AMS-trial 318 interaction; $p=0.2$ ).

320 Exercise performance (time to exhaustion; TTE)

321 Six days dietary nitrate supplementation had no effect on TTE in hypoxia $(\Delta 10[-103,123] \mathrm{s} ; p=0.9)$. Dietary 322 nitrate supplementation had no effect on heart rate, $\mathrm{SpO}_{2}$, or RPE at any isotime, or at exhaustion (Table 2).

324 Whether participants experienced AMS did not influence the effect of nitrate on exercise performance (TTE; 325 group-trial interaction; $p=0.9)$. Specifically, nitrate had no effect on TTE in AMS- $(\Delta 16[-115,148] \mathrm{s} ; p=0.8)$ or in $\mathrm{AMS}+(\Delta 10[-103,123] \mathrm{s} ; p=0.9)$. 
The primary findings of this study were that dietary nitrate supplementation did not reduce AMS severity, specifically high-altitude headache, or improve exercise performance in hypoxia. When assessed over the whole sample, nitrate had no effect on any physiological response to hypoxia. However, opposing effects of nitrate were observed in those with and without AMS. In participants who did not develop AMS (AMS-), nitrate decreased ventilation and improved $\mathrm{SpO}_{2}$ during exercise but this did not translate into reduced AMS symptoms or an improvement in exercise performance. In contrast, in AMS+, nitrate increased ventilation and $\mathrm{O}_{2}$ cost of exercise, headache and AMS severity, and sense of effort during submaximal exercise in 6 h hypoxia.

The increase in headache and AMS that occurred in the latter part of the trial was exacerbated by nitrate. It is clear this was not due to nitrate increasing exercise-induced headache, since there was no effect of nitrate on headache post exercise bout one or two that occurred earlier in the hypoxic exposure, before AMS was present. In those that developed AMS dietary nitrate induced a $26 \mathrm{~mm}$ increase in headache, which is of sufficient magnitude to have clinical relevance (23). This finding is in agreement with a previous study that reported greater headache at altitude after L-arginine supplementation (32). However it contrasts the null finding reported by Hennis and colleagues in the field (20); the only previous study to investigate the effects of chronic dietary nitrate supplementation on AMS. This difference in results is most likely due to differences in study design. Specifically, in Hennis and colleagues' study (20) biochemical confirmation of the nitrate supplementation was not performed and therefore it was not possible to determine if the nitrate supplementation was effective. This is of particular concern since the authors reported poor compliance. Further, as AMS is determined by subjective responses, the use of a non-taste matched placebo means placebo or nocebo effects cannot be dismissed. In contrast, we completed a double-blind placebo-controlled study, and report 100\% compliance to the supplementation protocol, with confirmation of successful blinding and increased plasma $\mathrm{NO}_{\mathrm{x}}$ in the nitrate trial.

When activated in the brain, NO directly stimulates the trigeminovascular system, responsible for headache pain sensation (3). In addition, an increase in cerebral blood flow and resultant increase in intracranial pressure can also result in trigeminovascular system activation, and is suggested to cause high-altitude headache and AMS (27). Therefore increasing the bioavailability of NO through dietary nitrate supplementation is likely to directly stimulate trigeminovascular afferents, and concurrently elevate hypoxia-induced cerebral vasodilation. This provides a possible mechanism for the increase in high-altitude headache and AMS observed herein. Further 
357

358

359

360

361

362

363

364

365

366

367

368

369

370

371

372

373

374

375

376

377

378

379

380

381

382

383

384

385

386

support for this explanation is provided by previous studies that have utilized NO-synthase inhibitors (e.g. LNMMA) (3) and artificial vasoconstriction (25) to successfully reverse headache.

Nitrate supplementation did not benefit exercise performance as assessed by time to exhaustion in acute hypoxia. This finding is in agreement with the only previous randomized controlled trial (RCT) to assess exercise performance in acute hypoxia $(<2 \mathrm{~h})$ following six days dietary nitrate supplementation (10). Bourdillon et al. (10) found dietary nitrate supplementation did not alter hypoxic pulmonary vasoconstriction or $15 \mathrm{~km}$ time trial performance in acute hypoxia $\left(\mathrm{F}_{\mathrm{I}} \mathrm{O}_{2}=0.11\right)$. A further novel approach of the current study was to examine exercise responses during $6 \mathrm{~h}$ hypoxic exposure after dietary nitrate supplementation. Contrary to our hypothesis, nitrate did not improve submaximal exercise performance even in AMS- where nitrate improved $\mathrm{SpO}_{2}$ during exercise (3\% after $5 \mathrm{~h}$ in hypoxia). Further, in those that developed AMS+, nitrate actually impaired exercise by increasing sense of effort; participants had to invest more effort to achieve the same exercise output in the nitrate trial, compared to placebo. In those that developed AMS (AMS+), nitrate also increased the $\mathrm{O}_{2}$ cost of fixed workload exercise, which may have been driven by an increase in ventilation. Increased ventilation is associated with greater respiratory muscle demand and dyspnea (1), which are important contributing factors of sense of effort (7). As exercising sense of effort, ventilation, and $\mathrm{O}_{2}$ cost of exercise were increased and present before AMS, nitrate should be considered the cause of the negative effects observed in AMS+, rather than an effect of AMS symptoms. In addition, the elevated AMS symptoms may have contributed to the increase in sense of effort in the final bout of exercise, as the increase in sense of effort was proportional to headache severity immediately before exercise. By the end of the final exercise bout sense of effort was increased by $54 \%$ in those who experienced AMS, equivalent to an entire verbal descriptor (from "moderate", to "somewhat strong"). Since the exercise typically completed at altitude is often long-duration and submaximal, this finding has great importance for those travelling to altitude for work and recreation as increased sense of effort is associated with poorer mood, and increased fatigue (39), which is an important risk factor at altitude (15).

This study is limited by the absence of a direct measure of cerebral blood flow to support the proposed mechanistic interpretation. However, the conclusion of cause and effect is supported by the use of a strong experimental design, and a theoretical explanation backed by a wealth of existing literature (27). This study was also limited by the duration of exposure ( $6 \mathrm{~h}$ ), and is therefore unable to conclude the effects of nitrate on physiological and perceptual responses with more chronic exposure to altitude, for example over many days or weeks. Another limitation of 
this study is that all participants were men, and thus the findings may not be applicable to women. In addition, the study design did not allow separation of the effects of nitrate in hypoxia per se from any interaction with exercise, although the effects of nitrate supplementation with exercise in normoxia have been studied in detail in previous literature (34).

In conclusion, dietary nitrate increases AMS symptom severity, specifically headache, and sense of effort during submaximal exercise, particularly in those who experience AMS. Therefore, dietary nitrate is not recommended as an AMS prophylactic or ergogenic aid in non-acclimatized individuals at altitude.

\section{ACKNOWLEDGMENTS}

The authors thank Beet It for providing the beverages used in this study at a reduced cost. We also thank Matteo Crotti, Morgan Gregory, and Harriet Shipp for their assistance with data collection, and Kevin Williams and Jason Edwards for their technical assistance.

\section{DISCLOSURES}

No conflicts of interest, financial or otherwise, are declared by the authors.

\section{AUTHOR CONTRIBUTIONS}

G.M.K.R., J.H.M., S.J.L., B.W., V.N., H.E.D., and S.J.O conception and design of research; G.M.K.R., J.H.M., L.J.W., S.J.L., B.W., V.N., K.A.H., R.B., H.E.D., and S.J.O performed experiments; G.M.K.R and L.J.W. analyzed data; G.M.K.R., J.H.M., L.J.W., S.J.L., K.A.H., R.B., and S.J.O interpreted results of experiments; G.M.K.R prepared figures; G.M.K.R., J.H.M., L.J.W., B.W., V.N., and S.J.O drafted manuscript; G.M.K.R., J.H.M., L.J.W., and S.J.O edited and revised manuscript; G.M.K.R., J.H.M., L.J.W., S.J.L., B.W., V.N., K.A.H., R.B., H.E.D., and S.J.O approved final version of manuscript. 
413 1. Amann M, Pegelow DF, Jacques AJ, Dempsey JA. Inspiratory muscle work in acute hypoxia influences 414 locomotor muscle fatigue and exercise performance of healthy humans. Am J Physiol Regul Integr Comp Physiol 293: 2036-2045, 2007.

2. Arnold JT, Oliver SJ, Lewis-Jones TM, Wylie LJ, Macdonald JH. Beetroot juice does not enhance altitude running performance in well-trained athletes. Appl Physiol Nutr Metab 40: 590-595, 2015. Ashina M, Lassen LH, Bendtsen L, Jensen R, Olesen J. Effect of inhibition of nitric oxide synthase on chronic tension-type headache: a randomised crossover trial. Lancet 353: 287-289, 1999. nitrate supplementation improves arterial endothelial function at high altitude: a double-blinded randomized controlled cross over study. Nitric Oxide 50: 58-64, 2015. play within world tourism? 2008.

Bärtsch P, Swenson ER. Clinical practice: Acute high-altitude illnesses. N Engl J Med 368: 2294-2302, 2013.

Bernhadt V, Wood HE, Moran RB, Babb TG. Dyspnea on exertion in obese men. Respir Physiol Neurobiol 185: 241-248, 2013.

Bland M. Determination of sample size. 4th ed. Oxford: Oxford University Press, 2015. $15-45,2001$.

10. Bourdillon N, Fan J, Uva B, Müller H, Meyer P, Kayser B. Effect of oral nitrate supplementation on

11. DiPasquale DM, Strangman GE, Harris NS, Muza SR. Hypoxia, hypobaria, and exercise duration Med 1: 546-551, 1995.

13. Eston R. Use of ratings of perceived exertion in sports. Int J Sports Physiol Perform 7: 175-182, 2012.

14. Ferguson SK, Hirai DM, Copp SW, Holdsworth CT, Allen JD, Jones AM, Musch TI, Poole DC. 
Effects of nitrate supplementation via beetroot juice on contracting rat skeletal muscle microvascular oxygen pressure dynamics. Respir Physiol Neurobiol 187: 250-255, 2013.

15. Firth PG, Zheng H, Windsor JS, Sutherland AI, Imray CH, Moore GWK, Semple JL, Roach RC, Salisbury RA. Mortality on Mount Everest, 1921-2006: descriptive study. Br Med J 337: a2654, 2008.

16. Fulco CS, Rock PB, Cymerman A. Maximal and submaximal exercise performance at altitude. Aviat Space Environ Med 69: 793-801, 1998.

17. Government of Nepal. Ministry of Culture Tourism \& Civil Aviation: Nepal Tourism Statistics 2013. 2013.

18. Govoni M, Jansson EA, Weitzberg E, Lundberg JO. The increase in plasma nitrite after a dietary nitrate load is markedly attenuated by an antibacterial mouthwash. Nitric Oxide 19: 333-337, 2008.

19. Hackett P, Roach RC. High altitude cerebral edema. High Alt Med Biol 5: 136-147, 2004.

20. Hennis PJ, Mitchell K, Gilbert-Kawai E, Bountziouka V, Wade A, Feelisch M, Grocott MP, Martin

21. Hoon MW, Johnson NA, Chapman PG, Burke LM. The effect of nitrate supplementation on exercise DS. Effects of dietary nitrate supplementation on symptoms of acute mountain sickness and basic

23. Kelly AM. The minimum clinically significant difference in visual analogue scale pain score does not

24. Kelly J, Vanhatalo A, Bailey SJ, Wylie LJ, Tucker C, List S, Winyard PG, Jones AM. Dietary nitrate

25. King AB, Robinson SM. Vascular headache of acute mountain sickness. Aerosp Med 43: 849-851, 1972. a placebo-controlled study. J Appl Physiol 110: 591-600, 2011.

27. Lawley JS, Levine BD, Williams MA, Malm J, Eklund A, Polaner DM, Subudhi AW, Hackett PH, 
Roach RC. Cerebral spinal fluid dynamics: effect of hypoxia and implications for high-altitude illness. $J$ Appl Physiol 120: 251-262, 2016.

28. Lawley JS, Macdonald JH, Oliver SJ, Mullins PG. Unexpected reductions in regional cerebral perfusion during prolonged hypoxia. J Physiol 595: 935-947, 2017.

29. Lawley JS, Oliver SJ, Mullins PG, Macdonald JH. Investigation of whole-brain white matter identifies altered water mobility in the pathogenesis of high-altitude headache. J Cereb blood flow Metab 33: 12861294,2013

30. Lawley JS, Oliver SJ, Mullins PG, Macdonald JH, Moore JP. Prolonged (9 h) poikilocapnic hypoxia (12\% O2) augments cutaneous thermal hyperaemia in healthy humans. Exp Physiol 99: 909-920, 2014.

31. Lundberg JO, Carlstörm M, Larsen FJ, Weitzberg E. Roles of dietary inorganic nitrate in cardiovascular health and disease. Cardiovasc Res 89: 525-532, 2011.

32. Mansoor JK, Morrissey BM, Walby WF, Yoneda KY, Juarez M, Kajekar R, Severinghaus JW, Eldridge MW, Schelegle ES. L-arginine supplementation enhances exhaled NO, breath condensate

33. Masschelein E, Van Thienen R, Wang X, Van Schepdael A, Thomis M, Hespel P. Dietary nitrate

34. McMahon NF, Leveritt MD, Pavey TG. The effect of dietary nitrate supplementation on endurance exercise performance in healthy adults: a systematic review and meta-analysis. Sport Med 47: 735-756,

35. Van Mil AHM, Spilt A, Van Buchem MA, Bollen ELEM, Teppema L, Westendorp RGJ, Blauw GJ.

36. Moran D, Epstein Y, Keren G, Laor A, Sherez J, Shapiro Y. Calculation of mean arterial pressure during exercise as a function of heart rate. Appl Hum Sci J Physiol Anthropol 14: 293-295, 1995. Nitric oxide mediates hypoxia-induced cerebral vasodilation in humans. J Appl Physiol 92: 962-966, 2002.

Muggeridge DJ, Howe CCF, Spendiff O, Pedlar C, James PE, Easton C. A single dose of beetroot juice enhances cycling performance in simulated altitude. Med Sci Sports Exerc 46: 143-150, 2014.

Roach RC, Maes D, Sandoval DA, Robergs RA, Icenogle M, Hinghofer-Szalkay H, Lium D, Loeppky JA. Exercise exacerbates acute mountain sickness at simulated high altitude. J Appl Physiol 88: $581-585,2000$. 
39. Rossetti GMK, Macdonald JH, Smith M, Jackson AR, Callender N, Newcombe HK, Storey HM, Willis S, van den Beukel J, Woodward J, Pollard J, Wood B, Newton V, Virian J, Haswell O, Oliver SJ. MEDEX2015: Greater sea-level fitness is associated with lower sense of effort during Himalayan trekking without worse acute mountain sickness. High Alt. Med. Biol. (2017). doi: 10.1089/ham.2016.0088.

40. Sampson J, Kobrick J. The Environmental Symptoms Questionnaire: revisions and new field data. Aviat Space Environ Med 51: 872-877, 1980.

41. Sampson JB, Kobrick JL, Johnson RF. Measurement of subjective reactions to extreme environments: the Environmental Symptoms Questionnaire. Mil Psychol 6: 215-233, 1994.

42. Shannon OM, Duckworth L, Barlow MJ, Deighton K, Matu J, Williams EL, Woods D, Xie L, Stephan BCM, Siervo M, O’Hara JP. Effects of Dietary Nitrate Supplementation on Physiological Responses, Cognitive Function, and Exercise Performance at Moderate and Very-High Simulated Altitude. Front Physiol 8: 401, 2017.

43. Siervo M, Lara J. Inorganic nitrate and beetroot juice supplementation reduces blood pressure in adults: a systematic review and meta-analysis. J Nutr 143: 818-826, 2013.

44. Stamler JS, Meissner G. Physiology of nitric oxide in skeletal muscle. Physiol Rev 81: 209-237, 2001.

45. Stevens J. Applied multivariate statistics for the social sciences. 4th ed. New Jersey: Lawrence Erlbaum Associates Inc., 2002.

46. Vanhatalo A, Fulford J, Bailey SJ, Blackwell JR, Winyard PG, Jones AM. Dietary nitrate reduces muscle metabolic perturbation and improves exercise tolerance in hypoxia. J Physiol 589: 5517-5528, 2011.

47. West JB, Schoene RB, Luks AM, Milledge JS. High Altitude Medicine and Physiology. 5th ed. Hoboken: Taylor and Francis, 2012.

48. Wilson MH, Davagnanam I, Holland G, Dattani RS, Tamm A, Hirani SP, Kolfschoten N, Strycharczuk L, Green C, Thornton JS, Wright AD, Edsell M, Kitchen ND, Sharp DJ, Ham TE, Murray AJ, Holloway CJ, Clarke K, Grocott MPW, Montgomery HE, Imray CHE. Cerebral venous system and anatomical predisposition to high-altitude headache. Ann Neurol 73: 381-389, 2013.

49. Wylie LJ, Kelly J, Bailey SJ, Blackwell JR, Skiba PF, Winyard PG, Jeukendrup AE, Vanhatalo A, Jones AM. Beetroot juice and exercise: pharmacodynamic and dose-response relationships. J Appl Physiol 115: 325-336, 2013. 
535 Figure 1. Overview of trial protocol. Supplementation was taken at the same time each day, $2.5 \mathrm{~h}$ before any 536 exercise tests. Participants completed a $6 \mathrm{~h}$ exposure to hypoxia on day five, and an acute hypoxic exercise to 537 exhaustion test on day six. All exercise intensities are set at a proportion of hypoxic $\dot{\mathrm{VO}}_{2 \max }$ reserve. Additional 538 physiological data was obtained by online gas analysis and a stress blood pressure system during exercise bout two. Filled black arrows indicate headache and AMS data included in the primary analysis.

Figure 2. High-altitude headache and AMS during $6 \mathrm{~h}$ in hypoxia $\left(\mathrm{F}_{1} \mathrm{O}_{2}=0.124\right)$ on day five. Grey shaded bars indicate submaximal exercise bouts $\left(40 \% \dot{\mathrm{V}}_{2 \max }\right.$ reserve). From 4 to $6 \mathrm{~h}$, in the whole sample, nitrate increased headache by visual analogue scale (VAS; Panel A), and tended to increase AMS-C score calculated from the Environmental Symptoms Questionnaire (Panel B). In AMS- (participants with AMS-C score $<0.7$ in both trials) dietary nitrate had no effect on headache (Panel C), or AMS-C (Panel D). In AMS+ (participants with AMS-C $\geq 0.7$ on placebo), nitrate increased headache (Panel E) and AMS-C (Panel F). *nitrate significantly higher than placebo $(p<0.05)$.

Figure 3. Sense of effort (RPE) and oxygen saturation $\left(\mathrm{SpO}_{2}\right)$ during submaximal exercise. In the whole sample, dietary nitrate increased RPE (Panel A), but had no effect on $\mathrm{SpO}_{2}$ (Panel B). In AMS- (participants with AMS-C score $<0.7$ in both trials), dietary nitrate had no effect on RPE (Panel C), but increased $\mathrm{SpO}_{2}$ during exercise bout three (Panel D). In AMS+ (participants with AMS-C $\geq 0.7$ on placebo), dietary nitrate increased RPE (Panel E), but had no effect on $\mathrm{SpO}_{2}($ Panel F). *nitrate significantly higher than placebo $(p<0.05)$.

Figure 4. Ventilation during submaximal exercise (exercise bout two). Dietary nitrate decreased ventilation during exercise bout two in AMS- (participants with AMS-C score $<0.7$ in both trials; Panel A), and increased ventilation in AMS+ (participants with AMS-C $\geq 0.7$ on placebo; Panel B). *nitrate significantly higher than placebo $(p<0.05)$.

559

Figure 5. Oxygen cost $\left(\dot{\mathbf{V}} \mathrm{O}_{2}\right)$ of submaximal exercise (exercise bout two). Dietary nitrate had no effect on $\dot{\mathrm{V}} \mathrm{O}_{2}$ during exercise bout two in AMS- (participants with AMS-C score $<0.7$ in both trials; Panel A), but increased $\dot{\mathrm{VO}}_{2}$ in AMS+ (participants with AMS-C $\geq 0.7$ on placebo; Panel B). *nitrate significantly higher than placebo ( $p$ $<0.05)$. 
565 Figure 6. Mean arterial pressure (MAP) during submaximal exercise (exercise bout two). Dietary nitrate

566 had no effect on MAP during exercise bout two in AMS- (participants with AMS-C score $<0.7$ in both trials;

567 Panel A), but increased MAP in AMS+ (participants with AMS-C $\geq 0.7$ on placebo; Panel B). Nitrate significantly 568 higher than placebo $(* p<0.05 ; * * p<0.01)$. 